Journal of Applied AnALysis

Vol. 14, No. 1 (2008), pp. 43-52

\title{
KOEBE DOMAINS FOR THE CLASSES OF FUNCTIONS WITH RANGES INCLUDED IN GIVEN SETS
}

\author{
L. KOCZAN and P. ZAPRAWA
}

Received March 12, 2007 and, in revised form, September 4, 2007

\begin{abstract}
In this paper we present a new method of determining Koebe domains. We apply this method by giving a new proof of the well-known theorem of A. W. Goodman concerning the Koebe domain for the class $T$ of typically real functions. We applied also the method to determine Koebe sets for classes of the special type, i.e. for $T^{M, g}=\{f \in T: f(\Delta) \subset M g(\Delta)\}, g \in T \cap S, M>1$, where $\Delta=\{z \in \mathbf{C}:|z|<1\}$ and $T, S$ stand for the classes of typically real functions and univalent functions respectively. In particular, we find the Koebe domains for the class $T_{M}$ of all typically real and bounded functions, and for the class $T(M)$ of all typically real functions with ranges in a given strip.
\end{abstract}

\section{INTRODUCTION}

A function $f$ analytic in the unit disk $\Delta=\{z \in \mathbf{C}:|z|<1\}$ is said to be typically real if it satisfies the condition $\operatorname{Im} z \operatorname{Im} f(z) \geq 0, z \in \Delta$. Let $T$ denote the class of typically real functions $f$ with normalization $f(0)=$

2000 Mathematics Subject Classification. Primary 30C25; Secondary 30C80.

Key words and phrases. Typically real functions, Koebe domain, subordination.

ISSN 1425-6908（C) Heldermann Verlag. 
$f^{\prime}(0)-1=0$ (see for example [5]). Let $S$ be the class of functions analytic and univalent in $\Delta$ with the same normalization as in $T$.

The following classes were considered in [3]

$$
T^{M, g}=\{f \in T: f \prec M g\}, g \in T \cap S, M>1 .
$$

Recall that a function $h$ is subordinated to a univalent function $H$, written $h \prec H$, if $h(0)=H(0)$ and $h(\Delta) \subset H(\Delta)$. Choosing

$$
g_{1}(z)=z \quad \text { and } \quad g_{2}(z)=\frac{1}{2} \log \frac{1+z}{1-z}, z \in \Delta,
$$

with the principal branch of logarithm, we obtain two important subclasses of $T$. Namely,

$$
T^{M, g_{1}}=\{f \in T:|f(z)|<M, z \in \Delta\}
$$

and

$$
T^{M, g_{2}}=\left\{f \in T:|\operatorname{Im} f(z)|<M \frac{\pi}{4}, z \in \Delta\right\},
$$

which are briefly denoted by $T_{M}$ and $\left.T(M)\right)$ respectively.

The relation

$$
T^{M, g}=\left\{M g\left(\frac{h(z)}{M}\right): h \in T_{M}\right\},
$$

which was established in [3], provides the formula connecting different classes of type $T^{M, g}$ as follows:

$$
T^{M, f}=\left\{M f\left(g^{-1}\left(\frac{h(z)}{M}\right)\right): h \in T^{M, g}\right\} .
$$

For this reason, instead of researching a class $T^{M, f}$ one can consider a class $T^{M, g}$. We apply this idea in order to obtain results in various classes $T^{M, g}$ as a consequence of related results in $T(M)$. Investigating $T(M)$ is possible thanks to the integral formula for this class. Moreover, extremal points as well as supporting points are known in $T(M)$ (see, [4]).

The main aim of this paper is to determine the Koebe set (usually called the Koebe domain) for $T^{M, g}$. Recall that for a given $A \subset T$, the Koebe set is defined by $\bigcap_{f \in A} f(\Delta)$ and is denoted by $K_{A}$.

From (3) it follows that

Lemma 1. Let $f, g \in T \cap S$. A set $D$ is the Koebe domain for $T^{M, g}$ iff $M \cdot f\left(g^{-1}(D / M)\right)$ is the Koebe domain for $T^{M, f}$.

\section{Proof.}

$$
w \in K_{T^{M, f}} \Longleftrightarrow w \in \bigcap_{F \in T^{M, f}} F(\Delta)
$$




$$
\begin{aligned}
& \Longleftrightarrow w \in \bigcap_{G \in T^{M, g}} \operatorname{Mf}\left(g^{-1}\left(\frac{G(\Delta)}{M}\right)\right) \\
& \Longleftrightarrow \forall_{G \in T^{M, g}} \quad w \in M f\left(g^{-1}\left(\frac{G(\Delta)}{M}\right)\right) \\
& \Longleftrightarrow \forall_{G \in T^{M, g}} \quad M g\left(f^{-1}\left(\frac{w}{M}\right)\right) \in G(\Delta) \\
& \Longleftrightarrow M g\left(f^{-1}\left(\frac{w}{M}\right)\right) \in K_{T^{M, f}} .
\end{aligned}
$$

\section{The Koebe domain for the Class $T$}

In 1977 Goodman determined the Koebe domain for $T$. Let

$$
r(\theta)=\frac{\pi \sin \theta}{4 \theta(\pi-\theta)}, \theta \in(0, \pi) .
$$

Theorem A ([1]). The Koebe domain for the class $T$ is a bounded domain, symmetric with respect to both axes of the complex plane. Its boundary in the upper half plane is given by the polar equation

$$
\varrho(\theta)= \begin{cases}r(\theta) & \text { for } \quad \theta \in(0, \pi), \\ \frac{1}{4} & \text { for } \quad \theta=0 \text { or } \theta=\pi .\end{cases}
$$

A new proof of Theorem A. Let $f \in T$ omit two values $\varrho e^{i \theta}$ and $\varrho e^{-i \theta}$, where $\varrho>0, \theta \in(0, \pi)$. A function

$$
\frac{f(z)-\varrho e^{i \theta}}{f(z)-\varrho e^{-i \theta}}
$$

is analytic in $\Delta$ and omits the points 0 and 1 . Hence a function

$$
h(z)=\frac{1}{i} \log \frac{f(z)-\varrho e^{i \theta}}{f(z)-\varrho e^{-i \theta}},
$$

with the branch of logarithm chosen in such a way that $h(0)=2 \theta$, is also analytic in $\Delta$.

From (5) we derive

$$
f(z)=\varrho \frac{e^{i \theta}-e^{-i \theta} e^{i h(z)}}{1-e^{i h(z)}} .
$$


We obtain $h(z) \neq 2 n \pi, n \in \mathbf{Z}$ because $1-e^{i h(z)} \neq 0$. This and the equality

$$
\operatorname{Im} f(z)=\frac{\varrho}{\left|1-e^{i h(z)}\right|^{2}}\left(1-e^{-2 \operatorname{Im} h(z)}\right) \sin \theta
$$

lead to $\operatorname{Im} z \operatorname{Im} h(z) \geq 0, z \in \Delta$. Since $h(0)=2 \theta, h(z) \neq 2 n \pi, n \in \mathbf{Z}$ and $h$ is typically real, we get $0<h(x)<2 \pi$ for real $x$.

Let

$$
H(z)=2 \theta+\frac{a \cdot z}{1-2 t z+z^{2}}, \quad \text { where } a=8(\pi-\theta) \frac{\theta}{\pi}, t=1-2 \frac{\theta}{\pi} .
$$

Then $H$ is univalent, $H(0)=2 \theta$ and $H(\Delta)=\mathbf{C} \backslash\{p \in \mathbf{R}: p \leq 0 \vee p \geq 2 \pi\}$. From the properties of $h$ and $H$ we conclude that the function $h$ is subordinated to a function $H$. Therefore $h(z)=H(w(z))$, where $w(z)=$ $H^{-1}(h(z))$. This gives $|w(z)| \leq|z|$ and

$$
\frac{2 \sin \theta}{\varrho}=h^{\prime}(0)=H^{\prime}(0) \cdot w^{\prime}(0)=a \cdot w^{\prime}(0) \leq a=8(\pi-\theta) \frac{\theta}{\pi} .
$$

Thus

$$
\varrho \geq \frac{\pi \sin \theta}{4 \theta(\pi-\theta)}
$$

with equality only in the case of $h=H$.

In the original proof of this theorem Goodman applied some properties of the so-called universal typically real functions. The existence of their inverse functions, which were defined on Riemann surfaces, played an essential role here. In his method all universal functions were generated by the function

$$
G(z)=\frac{1}{\pi} \tan \left(\frac{\pi z}{1+z^{2}}\right)
$$

The main advantage of our new method is that one can easily obtain extremal functions which correspond to boundary points of the Koebe domain.

In fact, the boundary points of the Koebe set for $T$ are related to functions

$$
F(z)=\frac{e^{i \theta}-e^{-i \theta} e^{i H(z)}}{1-e^{i H(z)}} \cdot \frac{\pi \sin \theta}{4 \theta(\pi-\theta)} .
$$

One can check that functions $F$ coincide with those found by Goodman in $[2]$, i.e.

$$
\frac{G\left(\frac{z+c}{1+c z}\right)-G(c)}{\left(1-c^{2}\right) G^{\prime}(c)} \text { with } \quad c=\frac{-\pi+2 \sqrt{\pi \theta-\theta^{2}}}{\pi-2 \theta}
$$

The method presented above may be applied to other classes that consist of functions with real coefficients. 


\section{The Koebe domain for the Class $T^{M, k}$}

At the beginning we discuss the class $T^{M, k}$ with $k(z)=z /(1-z)^{2}$. After that, we shall determine the Koebe domains for the classes $T^{M, g}$, where $M>1$ and $g$ is a typically real and univalent function. Observe that

$$
f \in T^{M, k} \quad \text { iff } \quad f \in T \wedge f(\Delta) \subset \mathbf{C} \backslash\left\{p \in \mathbf{R}: p \leq-\frac{M}{4}\right\} .
$$

From now on we assume the branch of argument $\arg \left(M+4 r e^{i \theta}\right)$ to be in $[0,2 \pi)$.

Theorem 1. A function $f \in T^{M, k}$ omits $r e^{i \theta}$ and $r e^{-i \theta}, \theta \in(0, \pi)$ if and only if there exists a function $h$ analytic in $\Delta$ such that

1. $h(0)=2 \theta, \quad h^{\prime}(0)=\frac{2 \sin \theta}{r}$,

2. $2 \arg \left(M+4 r e^{i \theta}\right)<h(x)<2 \pi$ for $-1<x<1$,

3. $\operatorname{Im} z \operatorname{Im} h(z) \geq 0, z \in \Delta$,

4. $f(z)=r \frac{e^{i \theta}-e^{-i \theta} e^{i h(z)}}{1-e^{i h(z)}}$.

\section{Proof.}

$(\Rightarrow)$

Let $f \in T^{M, k}$ and $f(z) \neq r e^{i \theta}, f(z) \neq r e^{-i \theta}$ for $\theta \in(0, \pi)$. Observe that $\left(f(z)-r e^{i \theta}\right) /\left(f(z)-r e^{-i \theta}\right)$ is an analytic function in $\Delta$ and omits 0 and 1 . There exists the function

$$
\log \frac{f(z)-r e^{i \theta}}{f(z)-r e^{-i \theta}}
$$

which we denote by $i h(z)$. The branch of logarithm is chosen to be $h(0)=$ $2 \theta$.

Hence

$$
f(z)=r \frac{e^{i \theta}-e^{-i \theta} e^{i h(z)}}{1-e^{i h(z)}} \text { and } \quad e^{i h(z)} \neq 1 .
$$

Therefore

$$
h(z) \neq 2 n \pi, n \in \mathbf{Z}
$$

Moreover,

$$
\operatorname{Im} f(z)=\frac{r}{\left|1-e^{i h(z)}\right|^{2}}\left(1-e^{-2 \operatorname{Im} h(z)}\right) \sin \theta
$$

which leads to

$$
\operatorname{Im} f(z)>0 \quad \text { iff } \quad \operatorname{Im} h(z)>0
$$


and

$$
\operatorname{Im} f(z)<0 \quad \text { iff } \quad \operatorname{Im} h(z)<0 .
$$

Consequently, $\operatorname{Im} z \operatorname{Im} h(z) \geq 0, z \in \Delta$. Then $h$ is typically real. From (7) and $h(0)=2 \theta$ we conclude that $h(x) \in(0,2 \pi)$ for $x \in(-1,1)$.

For $x \in(-1,1)$ the function $h(x)$ is increasing. It follows from univalence of typically real functions in the set $\left\{z \in \Delta:\left|1+z^{2}\right|>2|z|\right\}$, see [1]. For this reason and from $f(x)>-M / 4$ (by (6)) it follows that

$$
h(x)>\frac{1}{i} \log \frac{-M / 4-r e^{i \theta}}{-M / 4-r e^{-i \theta}},
$$

or equivalently $h(x)>2 \arg \left(M+4 r e^{i \theta}\right)$.

$(\Leftarrow)$

Let $h$ be an analytic function in $\Delta$ such that the conditions 1-4 of Theorem 1 are satisfied. With these assumptions (6) holds. Hence $\operatorname{Im} z \operatorname{Im} f(z) \geq 0$, $z \in \Delta, f$ is normalized by $f(0)=f^{\prime}(0)-1=0$ and $f(x)>-M / 4$ for $x \in(-1,1)$. It means that $f \in T^{M, k}$. By the definition of $h$ we know that $f$ omits $r e^{i \theta}$ and $r e^{-i \theta}$.

Theorem 2. The Koebe domain for the class $T^{M, k}$, where $k(z)=$ $z /(1-z)^{2}, M>1$, is a bounded domain, symmetric with respect to the real axis. Its boundary in the upper half plane is given by the polar equation $w=\varrho(M, \theta) e^{i \theta}, \theta \in[0, \pi]$, where

$$
\varrho(M, \theta)= \begin{cases}\frac{M}{4(M-1)}, & \theta=0 \\ r(M, \theta), & \theta \in(0, \pi) \\ \frac{1}{4}, & \theta=\pi,\end{cases}
$$

and $r=r(M, \theta)$ is the only solution of

$$
\arg \left(M+4 r e^{i \theta}\right)-\frac{4 r \theta(\pi-\theta)-\pi \sin \theta}{4 r(\pi-\theta)-\sin \theta}=0
$$

in $(r(\theta), \infty)$, and $r(\theta)$ is given by $(4)$.

Proof. Let $f \in T^{M, k}$ and $f(z) \neq r e^{i \theta}, f(z) \neq r e^{-i \theta}$ for $\theta \in(0, \pi), r>0$. We assume $r \geq r(\theta)$ because $T^{M, k} \subset T$. By Theorem 1 there exists a function $h$ satisfying the conditions $1-4$ of this theorem and hence

$$
f(z)=r \frac{e^{i \theta}-e^{-i \theta} e^{i h(z)}}{1-e^{i h(z)}} .
$$

Let

$$
H_{M, \theta}(z)=2 \theta+\frac{a \cdot z}{1-2 t z+z^{2}}
$$


where

$$
\begin{aligned}
& a=\frac{8(\pi-\theta)\left(\theta-\arg \left(M+4 r e^{i \theta}\right)\right)}{\pi-\arg \left(M+4 r e^{i \theta}\right)}, \\
& t=\frac{\pi-2 \theta+\arg \left(M+4 r e^{i \theta}\right)}{\pi-\arg \left(M+4 r e^{i \theta}\right)}, \\
& \theta \in(0, \pi), M>1 .
\end{aligned}
$$

One can check that $t \in(-1,1)$. The function $H_{M, \theta}$ is univalent, $H_{M, \theta}(0)=2 \theta$ and $H_{M, \theta}(\Delta)=\mathbf{C} \backslash\left\{p \in \mathbf{R}: p \leq 2 \arg \left(M+4 r e^{i \theta}\right) \vee p \geq 2 \pi\right\}$. From properties of $h$ and $H_{M, \theta}$ we obtain $h \prec H_{M, \theta}$. Therefore

$$
\frac{2 \sin \theta}{r}=h^{\prime}(0) \leq H_{M, \theta}^{\prime}(0)=a \text {. }
$$

Hence

$$
\frac{2 \sin \theta}{r} \leq \frac{8(\pi-\theta)\left(\theta-\arg \left(M+4 r e^{i \theta}\right)\right)}{\pi-\arg \left(M+4 r e^{i \theta}\right)}
$$

or equivalently

$$
\theta \frac{r-r(\theta)}{r-\frac{\theta}{\pi} r(\theta)}-\arg \left(M+4 r e^{i \theta}\right) \geq 0 .
$$

Let $M$ and $\theta$ be fixed. Let us denote by $g(r)$ the left hand side of (12). It is easily seen that $g(r(\theta))<0$. We shall prove that the equation $g(r)=0$ considered for $r \in[r(\theta), \infty)$ has only one solution.

We have

$$
\begin{aligned}
g^{\prime}(r)= & \frac{\sin \theta}{4\left(r-\frac{\theta}{\pi} r(\theta)\right)^{2}\left|M+4 r e^{i \theta}\right|^{2}}\left(16(1-M) r^{2}+8 M\left(\cos \theta+\frac{\sin \theta}{\pi-\theta}\right) r\right. \\
& \left.+M\left(M-\frac{\sin ^{2} \theta}{(\pi-\theta)^{2}}\right)\right) .
\end{aligned}
$$

Let $W(r)=a r^{2}+b r+c$ with $a=16(1-M), b=8 M(\cos \theta+\sin \theta /(\pi-\theta))$, $c=M\left(M-\sin ^{2} \theta /(\pi-\theta)^{2}\right)$. Since $a<0$ and $c>0$, the function $W(r)$ is zero in two points of different signs.

Let $r_{0}(\theta)$ be a positive zero of $W(r)$. We claim that $r_{0}(\theta)>r(\theta)$.

Indeed, if $r_{0}(\theta) \leq r(\theta)$ were satisfied then we would obtain $g^{\prime}(r)<0$ for $r>r(\theta)$. In this case it would be

$$
0>g(r(\theta))>\lim _{r \rightarrow \infty} g(r)=0,
$$

a contradiction.

We have actually proved that

$$
g^{\prime}(r)>0 \text { for } r \in\left[r(\theta), r_{0}(\theta)\right) \text { and } g^{\prime}(r)<0 \text { for } r>r_{0}(\theta) .
$$


From $\lim _{r \rightarrow \infty} g(r)=0$ and $g^{\prime}(r)<0$ for $r>r_{0}(\theta)$ it follows that

$$
g(r)>0 \text { for } r>r_{0}(\theta) .
$$

This and the inequalities $g(r(\theta))<0$ and $g^{\prime}(r)>0$ for $r \in\left[r(\theta), r_{0}(\theta)\right)$ lead to the conclusion that for $r \geq r(\theta)$ the equation $g(r)=0$ has only one solution which we shall denote by $r(M, \theta)$. Hence $g(r) \geq 0$ holds for $[r(M, \theta), \infty)$.

For the function

$$
F_{M, \theta}(z)=r(M, \theta) \frac{e^{i \theta}-e^{-i \theta} e^{i H_{M, \theta}}}{1-e^{i H_{M, \theta}}}, \theta \in(0, \pi)
$$

there is $r(M, \theta) e^{i \theta} \in \partial F_{M, \theta}(\Delta)$. Combining this with $r \geq r(M, \theta)$ we deduce that the points $r(M, \theta) e^{i \theta}, r(M, \theta) e^{-i \theta}, \theta \in(0, \pi)$ belong to the boundary of $K_{T^{M, k}}$. Consequently $K_{T^{M, k}}$ is a starlike set.

Let $\varrho(M, \theta) e^{i \theta}, \theta \in[0,2 \pi)$ be the polar equation of this boundary. Hence

$$
\varrho(M, \theta)=r(M, \theta), \theta \in(0, \pi) .
$$

Dividing (12) by $\theta$ and taking the limit as $\theta$ tends to 0 from the right we get

$$
\frac{r-1 / 4}{r}-\frac{4 r}{M+4 r} \geq 0
$$

since

$$
\begin{aligned}
\lim _{\theta \rightarrow 0^{+}} \frac{\arg \left(M+4 r e^{i \theta}\right)}{\theta} & =\lim _{\theta \rightarrow 0^{+}} \operatorname{Im} \frac{4 i r e^{i \theta}}{M+4 r e^{i \theta}}=\lim _{\theta \rightarrow 0^{+}} \frac{4 r(M \cos \theta+4 r)}{\left|M+4 r e^{i \theta}\right|^{2}} \\
& =\frac{4 r}{M+4 r}
\end{aligned}
$$

As a result, (12) gives $r \geq M /(4(M-1))$. Hence $\varrho(M, 0)=M /(4(M-1))$.

Writing (10) in the form

$$
r \geq \frac{\left(\pi-\arg \left(M+4 r e^{i \theta}\right)\right) \sin \theta}{4(\pi-\theta)\left(\theta-\arg \left(M+4 r e^{i \theta}\right)\right)}
$$

and taking the limit as $\theta$ tends to $\pi$ from the left we obtain $r \geq 1 / 4$.

Indeed, for $f \in T^{M, k}$ we have $f(x)>-M / 4$ while $x \in(-1,1)$. Therefore $f$ for negative $x$ takes values from $(-\infty, b]$, where $b>-M / 4$. Thus $\lim _{\theta \rightarrow \pi^{-}} \arg \left(M+4 r e^{i \theta}\right)=0$.

The inequality $r \geq 1 / 4$ means that $\varrho(M, \pi)=1 / 4$. 
We have proved that the boundary of the Koebe domain for $T^{M, k}$ in the upper half plane is given in the form $w=\varrho(M, \theta) e^{i \theta}, \theta \in[0, \pi]$, where

$$
\varrho(M, \theta)= \begin{cases}\frac{M}{4(M-1)}, & \theta=0 \\ r(M, \theta), & \theta \in(0, \pi) \\ \frac{1}{4}, & \theta=\pi .\end{cases}
$$

Since Koebe domains for classes of functions with real coefficients are symmetric with respect to the real axis, the proof is complete.

Observe that the extremal functions in the cases of $\theta=0$ and $\theta=\pi$ are of the form

$$
F_{1}(z)=\frac{z}{1-2 z\left(\frac{2}{M}-1\right)+z^{2}} \quad \text { and } \quad F_{2}(z)=\frac{z}{(1+z)^{2}} .
$$

These functions belong to $T^{M, k}$ and satisfy

$$
F_{1}(1)=\frac{M}{4(M-1)} \quad \text { and } \quad F_{2}(-1)=-\frac{1}{4}
$$

By Lemma 1 and Theorem 2,

Corollary 1. The Koebe domain for the class $T_{M}$ is a bounded domain, symmetric with respect to both axes of the complex plane. Its boundary in the upper half plane is given by the parametric equation

$$
w=\frac{2 \varrho(M, \theta) e^{i \theta}}{\frac{2}{M} \varrho(M, \theta) e^{i \theta}+1+\sqrt{\frac{4}{M} \varrho(M, \theta) e^{i \theta}+1}}, \quad \theta \in[0, \pi],
$$

where $\varrho(M, \theta)$ is given by $(8)$.

Corollary 2. The Koebe domain for the class $T(M)$ is a bounded domain, symmetric with respect to both axes of the complex plane. Its boundary in the upper half plane is given by the parametric equation

$$
w=\frac{M}{4} \log \left(\frac{4}{M} \varrho(M, \theta) e^{i \theta}+1\right), \theta \in[0, \pi],
$$

where $\varrho(M, \theta)$ is given by $(9)$. 
Observe that the parametric complex equation (16) can be rewritten, using (10), in the form

$$
\left\{\begin{array}{l}
x=\frac{M}{8} \log \left(1+\frac{8}{M} \varrho(M, \theta) \cos \theta+\frac{16}{M^{2}} \varrho^{2}(M, \theta)\right) \\
y=\frac{M}{4} \frac{4 \varrho(M, \theta) \theta(\pi-\theta)-\pi \sin \theta}{4 \varrho(M, \theta)(\pi-\theta)-\sin \theta}
\end{array}, \theta \in[0, \pi] .\right.
$$

\section{References}

[1] Golusin, G., On typically-real functions (Russian), Mat. Sb. 27(69) (1950), 201-218.

[2] Goodman, A. W., The domain covered by a typically real function, Proc. Amer. Math. Soc. 64 (1977), 233-237.

[3] Koczan, L., On classes generated by bounded functions, Ann. Univ. Mariae Curie Sklodowska Sect. A 52(2) (1998), 95-101.

[4] Koczan, L., Szapiel, W., Extremal problems in some classes of measure (IV): typically real functions, Ann. Univ. Mariae Curie Sklodowska Sect. A 43 (1989), 55-68.

[5] Rogosinski, W. W., Über positive harmonische Entwicklungen und tipisch-reelle Potenzreichen (German), Math. Z. 35 (1932), 93-121.

LEOPOLD KOCZAN

DEPARTMENT OF APPLIED

MATHEMATICS

LUBLIN UNIVERSity OF TECHNOLOGY

NADBYSTRZYCKA 38 D

20-618 Lublin, Poland

E-MAIL: L.KOCZAN@POLLUB.PL
PAWE⿺ ZAPRAWA Department of Applied

MATHEMATICS

LUBLIN UNIVERSiTY OF TECHNOLOGY

NAdBystrzyCKA 38 D 20-618 Lublin, Poland E-MAIL: P.ZAPRAWA@POLLUB.PL 